\title{
Predicting Electricity Consumption Based on Optimized Model of $\operatorname{GM}(\mathbf{1 , 1})$
}

\author{
Zhang Zheng $^{1}$, Yang Shanlin ${ }^{1}$, Liu Huizhou ${ }^{2}$ and Yu Bengong ${ }^{1}$ \\ ${ }^{1}$ School of Management, Hefei University of Technology, Hefei, 230009, P.R. China \\ ${ }^{2}$ Tongling Power Supply Company, Anhui Electric Power Corporation, Tongling, \\ 244000, P.R. China \\ zhzheng99@sina.com
}

\begin{abstract}
Optimized GM(1,1) model based on least absolute criteria is proposed in this paper. Since the initial condition of original GM(1,1) model is not very suitable, we use the modified latest data which generating from the accumulative generating operation os the new initial condition. And the least absolute criteria is applied instead of least square criteria to improve the stability and prediction accuracy of GM(1,1) model. Then the particle swarm optimization is adapted to the parameters optimization. At the end, the optimized $G M(1,1)$ model is used to predict the whole social electricity consumption of China and the result shows its prediction accuracy is better than the original modeh and the GM(1,1) model with latest initial condition.
\end{abstract}

Keywords: Prediction; $G M(1,1)$ model, Least absolute criteria, PSO, Initial condition

\section{Introduction}

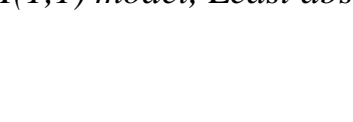

With the rapid econoric development in China, electricity demand increases with incredible speed. In the roduction and living of society, electricity rationing still happens and it does significant influence to enterprise's production activities. The electricity is an indispensable part of people's daly life. But if produce electricity too much, it will cause a great waste of resources and serious environmental pollution. Thus, precise prediction of electricity consunption will make production; supply and sales of power system achieve dynamic balance. It will also guarantee economic development and increase the economic benefit and social benefit

There are many methods used to predict electricity consumption and some certain results have achieved. Zafer and Lester[1] applied the structural time series technique to estimate an industrial electricity demand function for Turkey and this technique also uncovered the electricity Underlying Energy Demand Trend (UEDT) for the Turkish industrial sector to identify the size and significance of the price and industrial value added (output) elasticities. Azadeh et al., [2] used a hybrid adaptive network based fuzzy inference system (ANFIS), compater simulation and time series algorithm to estimate and predict electricity consumption estimation. Then the proposed method is applied to the monthly electricity consumption in Iran from 1995 to 2005.Kavaklioglu [3] adopted the Support Vector Regression (SVR) model to predict Turkey's electricity consumption and achieved good results. Geoffrey and Kelvin [4] compared the three methods of traditional regression analysis, decision tree and neural networks for the prediction of electricity energy consumption.

Grey system theory was first proposed by Deng [5] in 1982 and grey model is one of the most popular approaches in prediction field. A lot of experts and scholars take part in this 
research and apply to various fields of economy, environment and society. In these grey models, $\operatorname{GM}(1,1)$ model is the most studied model. In the meanwhile, the initial condition, background value etc., have great influence to prediction results from $\operatorname{GM}(1,1)$ modeling process. Pretty of researches concentrate on them in order to enhance the precision of prediction. Xiao et al., [6] studied GM $(1,1)$ model by using the thought of matrix analysis and put forward extension form GGM $(1,1)$ model based on the fractional order accumulated generating. Zhou and He [7] pointed out that there are two shortcomings in grey model, the homogeneous-exponent simulative deviation in GM $(1,1)$ model, and the unequal conversion between the original and white equations in $\operatorname{DGM}(1,1)$ model. They proposed a novel model based on GM $(1,1)$ and DGM $(1,1)$ models. Huang et al., [8] proposed an improved error GM $(1,1)$ model to predict cultivated land in Yiyang and the results show that the model had high prediction accuracy. Tien [9] did some research in model $\operatorname{GM}(1, n)$ and applied the algorithm of $\operatorname{GMC}(1, n)$ to explain the existing $\operatorname{GM}(1, n)$ model is incorrect.

On the one hand, lots of researches focus on initial condition of $\operatorname{GM}(1,1)$ model Zhang et al., [10] point out that $x^{(1)}(1)$ shouldn't be limited as the only know condition when forming the prediction formula. He used $x^{(0)}(k)$ which could make the model of highest accuracy as the initial condition. Wang et al., [11] take the first item and the last item of a sequence generated from applying the first-order accumulati generation operator as the new initial condition. Shih et al., [12] replace the initial value of grey differential equation to the latest point to enhance its accuracy.

On the other hand, lots of researches focus on background value of $\operatorname{GM}(1,1)$ model. Wang et al., [13] find that using an integral instead of riginal background value is more adaptive to whitenization equation. Wang[14] constrocts the background value by using Gaussian quadrature formula and improves the prediction aceuracy.

In this paper, we first introduce the original $\operatorname{GM}(1,1)$ model. Then we take the latest point as the initial condition to improve the accuracy of prediction. In addition, least absolute criteria is applied to the GM(1,1) model instead of least square criteria. It enhance the stability and prediction accuracy of GM(1) model. And particle swarm optimization (PSO) is used to parameters optimization of $\operatorname{GM}(1,1)$ model. At last, the whole social electricity consumption of China is adopted as the example and the results show better than the original model and the GM(1,1) model with latest initial condition.

\section{Original GM $(1,1)$ Model}

There are many advantages in grey prediction method. This method can provide a high degree of accuracy when the information is uncertain and the sample data are less. The main process is as follows:

Assume a non-negative original sequence is that

$X^{(0)}=\left\{\infty^{(0)}(1), x^{(0)}(2), \cdots, x^{(0)}(n)\right\}$

and the first order accumulative generating operation of $X^{(0)}$ is shown as follows

$$
X^{(1)}=\left\{x^{(1)}(1), x^{(1)}(2), \cdots, x^{(1)}(n)\right\}
$$

Where

$$
x^{(1)}(k)=\sum_{i=1}^{k} x^{(0)}(i), k=1,2, \cdots, n
$$


Due to the sequence $x^{(1)}(k)$ possesses exponential growth rule, and the solution of first order differential equation is just exponential form. So the sequence $x^{(1)}(k)$ can meet the form which is the solution of one order differential equation. The differential equation is that

$\frac{d x^{(1)}}{d t}+a x^{(1)}=u$

Eq.(4) is called whitened differential equation of $\operatorname{GM}(1,1)$. $a$ is called developing coefficient. It reflects the trend of development of $x^{(1)}$ and $x^{(0)} \cdot u$ is called grey action quantity[15]. It reflects the relationship between the data.

Discrediting the Eq.(4), we have

$\Delta\left(x^{(1)}(k)\right)+a z^{(1)}(k)=u$

Where

$\Delta\left(x^{(1)}(k)\right)=x^{(1)}(k)-x^{(1)}(k-1)=x^{(0)}(k)$

$z^{(1)}(k)=\frac{1}{2} x^{(1)}(k)+\frac{1}{2} x^{(1)}(k-1)$

Eq.(5) is a grey differential equation, and also called $\operatorname{GM}(1,1)$ model. $z^{(1)}(k)$ is called background value.

Let

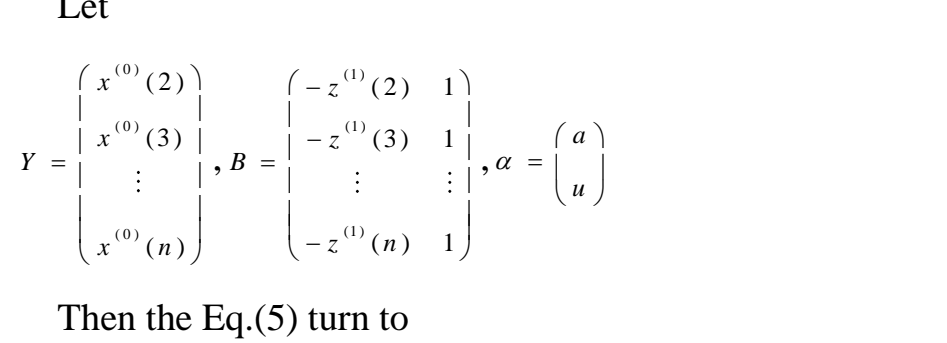

Then the Eq.(5) turn to $Y=B \alpha$

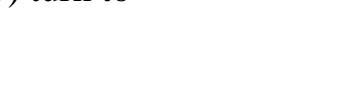

In terms of least square method, we have

$$
\min _{a, u} \sum_{k=2}^{n}\left[x^{(0)}(k)-\left(-a z^{(1)}(k)\right]^{2}\right.
$$

And

$\hat{\alpha}=\left(\begin{array}{l}\hat{a} \\ \hat{u}\end{array}\right)=(B)^{-1} B^{T} Y$

Substituting the Eq.(10) into the Eq.(4), the time response function of the whitened differential equation is got. That is

$x^{(1)}(t)=\left(x^{(1)}(1)-\frac{\hat{u}}{\hat{a}}\right) e^{-\hat{a}(t-1)}+\frac{\hat{u}}{\hat{a}}$

Then, the time response function of grey differential equation is

$$
\hat{x}^{(1)}(k+1)=\left(x^{(0)}(1)-\frac{\hat{u}}{\hat{a}}\right) e^{-\hat{a} k}+\frac{\hat{u}}{\hat{a}}
$$


$k=1,2, \cdots, n$

By inverse accumulative generating operation, the recovered value is shown below

$$
\hat{x}^{(0)}(k+1)=\hat{x}^{(1)}(k+1)-\hat{x}^{(1)}(k)=\left(1-e^{\hat{a}}\right)\left(x^{(0)}(1)-\frac{\hat{u}}{\hat{a}}\right) e^{-\hat{a} k}
$$

\section{Optimized GM(1,1) Model}

\subsection{Optimization of Initial Condition}

Through original $\operatorname{GM}(1,1)$ model, the initial condition which make the time response function be established is $x^{(1)}(1)=x^{(0)}(1)$. But the fitting curve does not necessarily pass through the initial data point for prediction. The point $x^{(0)}(1)$ is an old data; it doesn thave close relationship with the future data. And the latest data have more information and the relationship with future data is close. Thus, let the initial condition be

$\hat{x}^{(1)}(n)=c x^{(1)}(n)$

The time response function of grey differential equation becomes

$\hat{x}^{(1)}(k+1)=\left(c x^{(1)}(n)-\frac{\hat{u}}{\hat{a}}\right) e^{-\hat{a}(k-n+1)}+\frac{\hat{u}}{\hat{a}}$

$k=1,2, \cdots, n$

and by inverse accumulative generating operation, the recovered value becomes

$\hat{x}^{(0)}(k+1)=\hat{x}^{(1)}(k+1)-\hat{x}^{(1)}(k)=\left(e^{-\hat{a}}-1\right)\left(e x^{(1)}(n)-\hat{u} e^{-\hat{a}(k-n)}\right.$

\subsection{PSO-GM(1,1) Model based on Leást Absolute Criteria}

The GM(1,1) model uses least square method to obtain parameters from Eq.(9).and Eq.(10). Least square method is widely used in regression analysis. It is easy to calculate and has good anatical performance. But the error will be expanded when the sequence has singular point by least square method and the accuracy of prediction will be decrease.

The least absolute criteria use the first power of error instead of the quadratic error. Thus the singular points have less influence on the model and error will not be expanded. So the least absolute method has better stability than least square method, and also has better performance on statistics. The least absolute criteria use absolute error to describe the deviation, that is

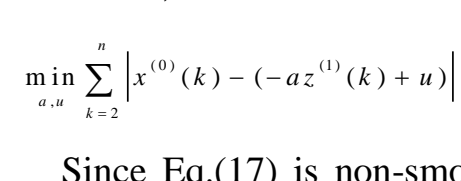

Since Eq.(17) is non-smooth and non- analytical, it is difficult to solve the parameters. Thus intelligent optimization algorithms are considered. In this paper, particle swarm optimization is adopted to optimize parameters.

Kennedy and Eberhart [16] proposed particle swarm optimization(PSO) in 1995. At first some scientists studied the behavior of a flock of birds. They found that a huge group of birds could change direction, scatter and restructure group, etc., when they were flying and focused on the processing of individual distance. Then Kennedy and Eberhart amended the model, 
making particles fly to the solution space and land on the optimal solution. And the flow chart of PSO algorithm is shown in Figure 1.

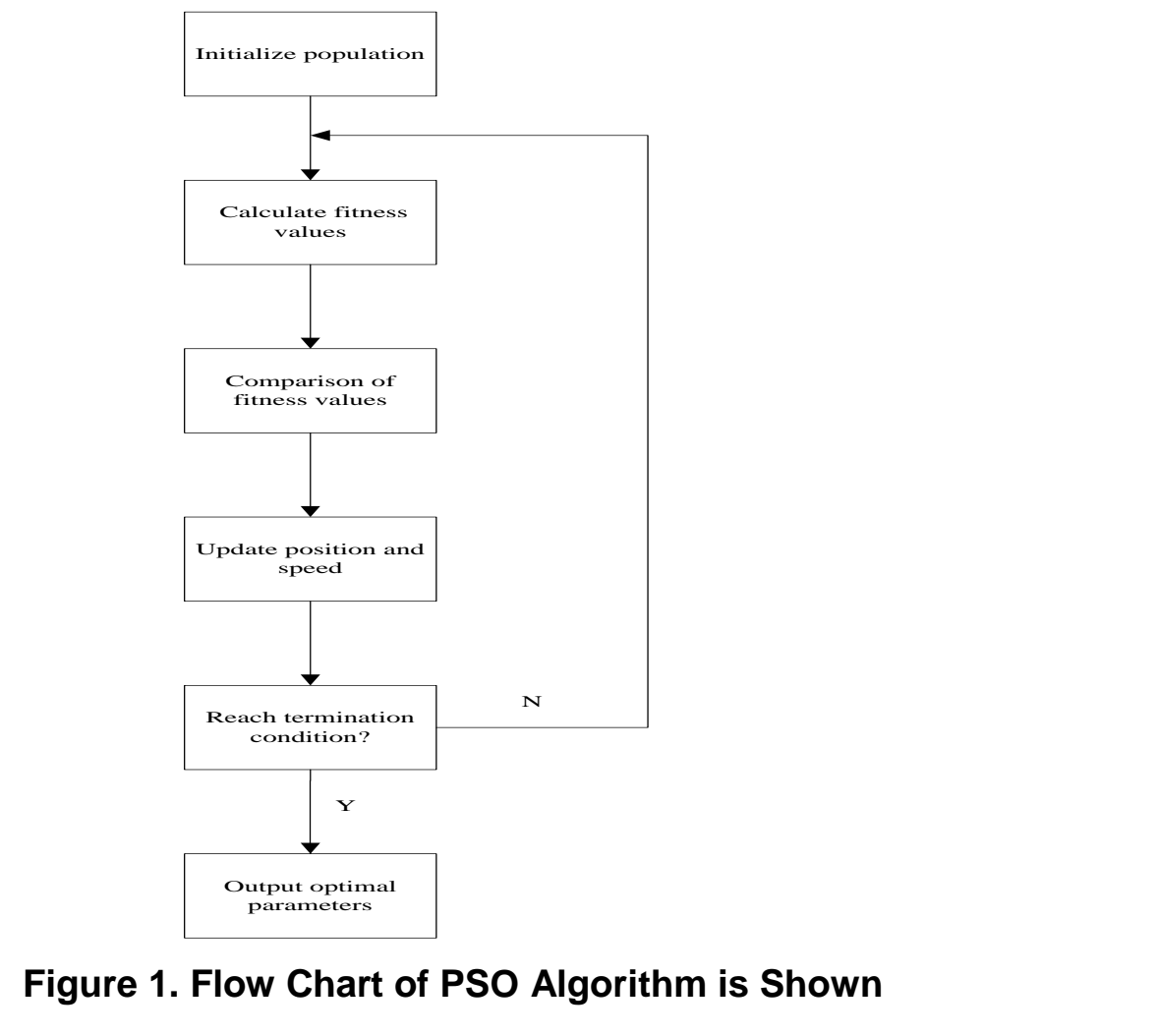

The principle of PSO is as follows. Set the number of particles is $N$. The particles fly in the d-dimensional space an a certain speed. Every particle considers the historical best point of itself and the historical best point of group when they search in the space. And update the position of particles on the basis of h/storical best point of itself and group.
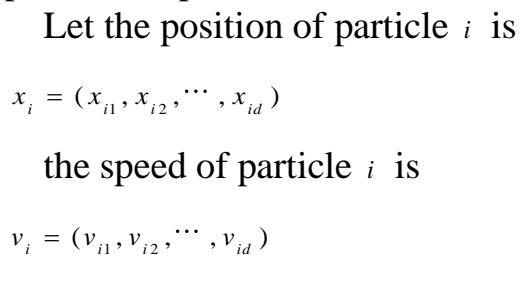

the historicar best point of particle $i$ is

$p_{i}=\left(p_{i 1}, R_{2}, \cdots, p_{i d}\right)$

the historical best point of group is

$p_{g}=\left(p_{g_{1}}, p_{g_{2}}, \cdots, p_{g d}\right)$

The position and speed of particle change rule is as follows

$$
\begin{aligned}
& v_{i d}^{k+1}=\omega v_{i d}^{k}+c_{1} \eta_{1}\left(p_{i d}^{k}-x_{i d}^{k}\right)+c_{2} \eta_{2}\left(p_{g d}^{k}-x_{i d}^{k}\right) \\
& x_{i d}^{k+1}=x_{i d}^{k}+v_{i d}^{k+1}
\end{aligned}
$$


Where $c_{1}$ and $c_{2}$ are called learning factor. Generally, $c_{1}=c_{2}=2 . \eta_{1}$ and $\eta_{2}$ are random number which are subjected to uniform distribution $U[0,1]$. The maximum speed of particles is $V_{\max }$.In order to make the particle have better exploration ability at the early stage and have better development ability at the later stage, the weight coefficient is set to

$\omega=\omega_{i}=\omega_{\max }-\frac{\omega_{\max }-\omega_{\min }}{\text { Iter }_{-} \max } * i$

Where Iter $_{-} \mathrm{m}_{\text {ax }}$ is maximum iterations. $\omega_{\max }=0.9, \omega_{\min }=0.4$.

The optimized initial condition of PSO-GM(1,1) algorithm based on least absolute criteria is as follows:

Step 1: The population size of particles is set to $N=50$ and The particle is set to twodimensional vector $x=\left(x_{1}, x_{2}\right) \cdot\left(x_{1}, x_{2}\right)$ is stand for $(a, u)$. Initialize position and speed of particles randomly.

Step 2: Calculate the fitness values of every particles. The fitness function i

$F=\sum_{k=2}^{n}\left|x^{(0)}(k)-\left(-a z^{(1)}(k)+u\right)\right|$

Step 3: For each particle, compared its fitness value with historical best fitness value of individual. If the current fitness value of individual is lowereit is set to the historical best fitness value of individual particle and the individual historical best position is updated by the current position.

Step 4: For each particle, compared itshistorical best litness value with the group historical best fitness value. If the historical fitness value of individual is lower, it is set to the historical fitness value of group and the group historical best position is updated by the individual best position.

Step 5: Update the speed and position of particles according to Eq.(18) and Eq.(19).

Step 6: Determine whether the algorithn reaches termination condition. If doesn't, turn to Step 2. The termination Condition is Ite - $\max =1000$.

After the process above, the optimal parameters $a$ and $u$ will be found. Then we need to solve parameter of initial condition. Hence, the same method is adopted while the fitness function is

$$
F^{*}=\sum_{k=2}^{n} \mid x^{(1)}(k)-\hat{x}^{(1)}(k)
$$

Then PSO algorithm is also used to find the optimal parameter $c$.

\section{Mode Construction and Prediction}

We construct the optimized $\operatorname{GM}(1,1)$ model based on least absolute criteria by Matlab and the data is the whole social electricity consumption of China from 1980 to 2010. Here the data from 1980 to 2006 are used to construct the model. The others which are from 2007 to 2010 are used to predict. Then the original $\operatorname{GM}(1,1)$ model, $\operatorname{GM}(1,1)$ model with latest initial condition and optimized $\operatorname{GM}(1,1)$ model proposed in this paper are established for comparative analysis. The results are shown as follows. 


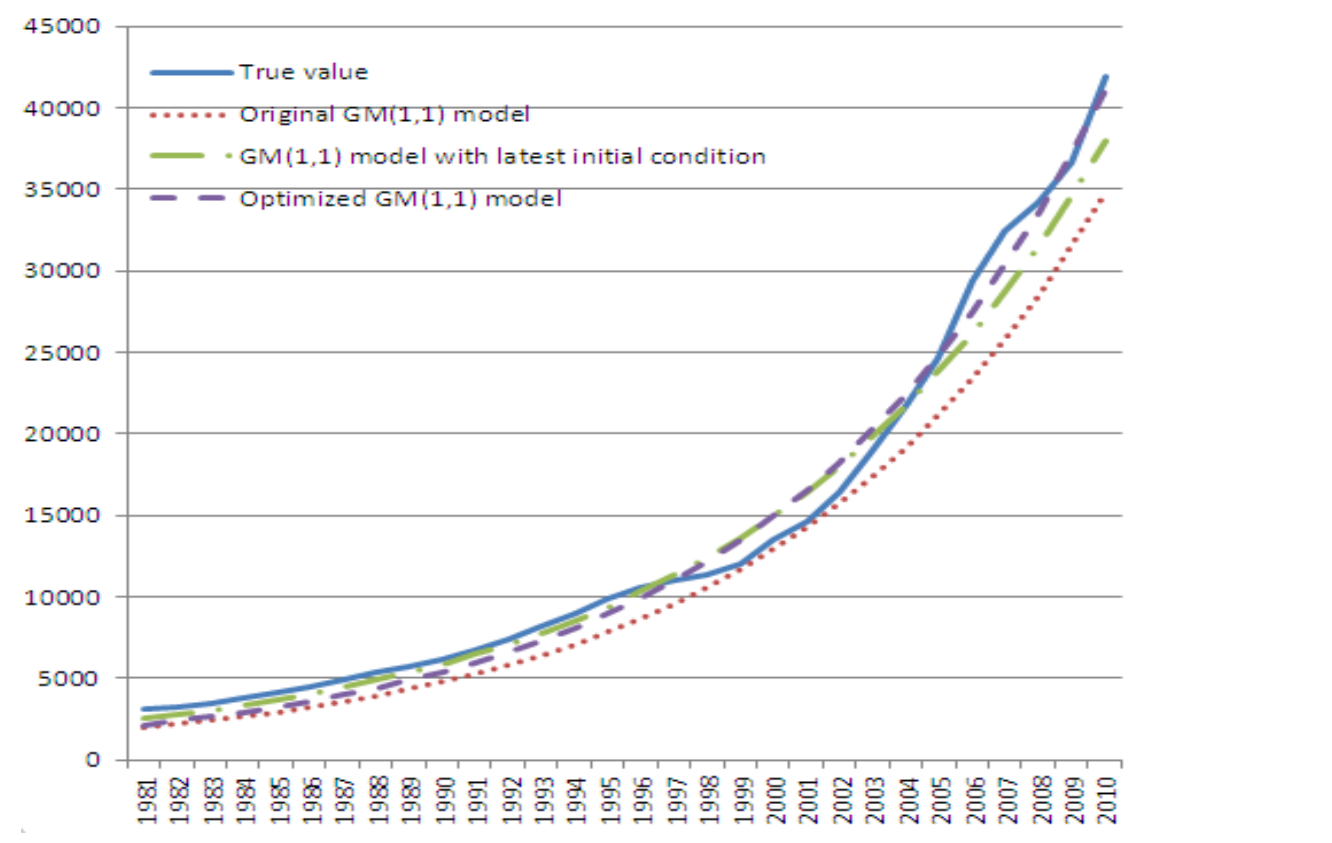

Figure 2. The True Value and the Prediction Curves of Different Methods (Hundred Minlion KWH)

Table 1. The Prediction Results of DifferentMethods from 1981to 2006

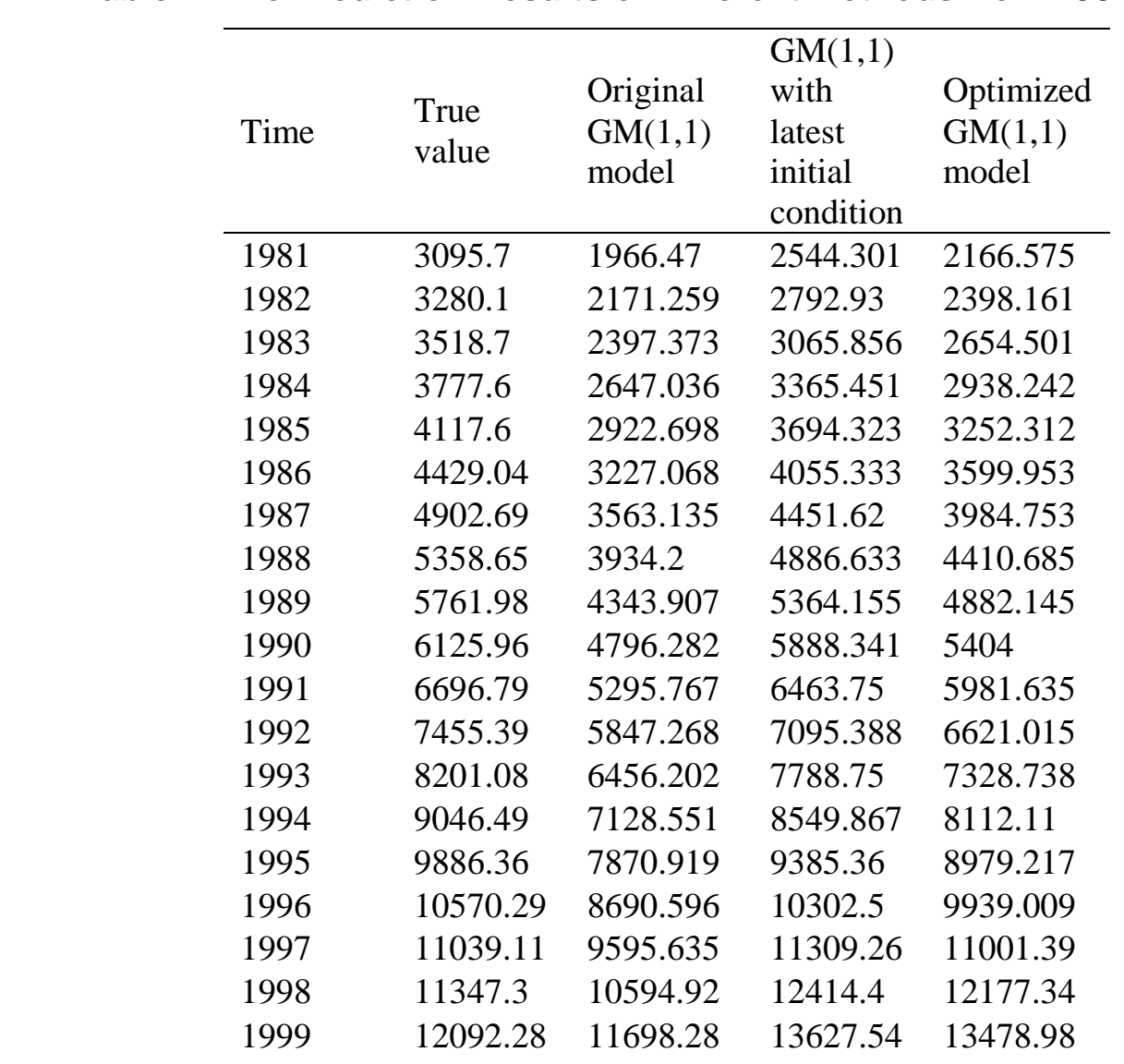




\begin{tabular}{lllll}
2000 & 13466.22 & 12916.54 & 14959.22 & 14919.75 \\
2001 & 14682.51 & 14261.67 & 16421.04 & 16514.53 \\
2002 & 16386.28 & 15746.88 & 18025.7 & 18279.78 \\
2003 & 18891.21 & 17386.76 & 19787.18 & 20233.71 \\
2004 & 21761.3 & 19197.41 & 21720.78 & 22396.5 \\
2005 & 24688.54 & 21196.63 & 23843.34 & 24790.47 \\
2006 & 29368 & 23404.05 & 26173.31 & 27440.34 \\
\hline
\end{tabular}

Table 2. The Total Prediction Accuracy of Different Methods

\begin{tabular}{|c|c|c|c|}
\hline & $\begin{array}{l}\text { Original } \\
\text { GM }(1,1) \\
\text { model }\end{array}$ & $\begin{array}{l}\mathrm{GM}(1,1) \\
\text { with latest } \\
\text { initial } \\
\text { condition }\end{array}$ & $\begin{array}{l}\text { Optimized } \\
\text { GM }(1,1) \\
\text { model }\end{array}$ \\
\hline RMSE & 2876.4973 & 1490.3345 & 1078.7505 \\
\hline MAPE & 0.1892229 & 0.0804278 & 0.1158562 \\
\hline $\mathrm{a}$ & -0.093235 & -0.09 & \\
\hline $\mathrm{u}$ & 1963.4871 & 196 & \\
\hline $\mathrm{c}$ & -- & & 1.0044508 \\
\hline
\end{tabular}

Where $R M S E=\sqrt{\frac{1}{n} \sum_{i=1}^{n}\left(\hat{y}_{i}-y_{i}\right)^{2}}$, MAPE $=1$
The initial condition of $\mathrm{GM}(1,1)$ model with latest initial condition is $\hat{x}^{(1)}(n)=x^{(1)}(n)$.

From Figure 2, these three methods all have a good fitting precision in early years. However, the fitting and prediction values of original $\mathrm{GM}(1,1)$ model become more and more far away from true values with the passage of time. From Table 2, the root mean square error (RMSE) and mean absolute percentage error (MAPE) of original $\operatorname{GM}(1,1)$ model are the biggest in three methods. In addition, RMSE of optimized $\operatorname{GM}(1,1)$ model proposed in this paper are lower than GM $(1,1)$ model yith latest initial condition, but MAPE of optimized $\operatorname{GM}(1,1)$ model proposed in this paper are higher than $\operatorname{GM}(1,1)$ model with latest initial condition. It only shows th at the $\operatorname{GM}(1,1)$ model with latest initial condition has better performance infitting precision to some extent. It doesn't mean that this method is better in prediction.

\section{Table 3. Prediction Value of Different Methods}

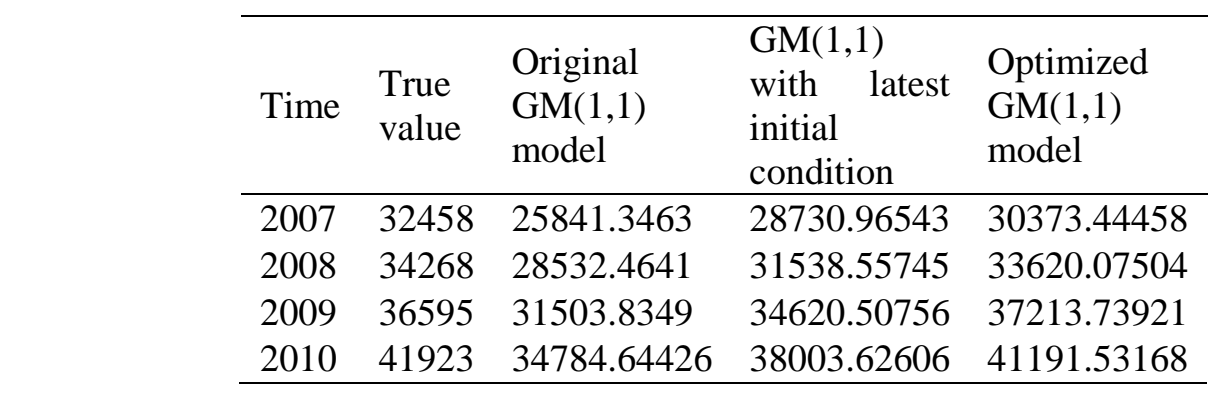


Table 4. Prediction Accuracy of Different Methods

\begin{tabular}{llll}
\hline & $\begin{array}{l}\text { Original } \\
\text { GM(1,1) } \\
\text { model }\end{array}$ & $\begin{array}{l}\text { GM(1,1) } \\
\text { with latest } \\
\text { initial } \\
\text { condition }\end{array}$ & $\begin{array}{l}\text { Optimized } \\
\text { GM(1,1) } \\
\text { model }\end{array}$ \\
\hline RMSE & 6195.81639 & 3185.936117 & 1191.9587 \\
MAPE & 0.170155155 & 0.085480337 & 0.029371599 \\
\hline
\end{tabular}

The prediction values and prediction accuracy of three methods are shown in Table 3 and Table 4. According to RMSE and MAPE, optimized GM(1,1) model based on least absolute criteria is the best of three methods in prediction accuracy on the whole.

Table 5. Relative Error of Different Methods

\begin{tabular}{llll}
\hline & Original & \multicolumn{2}{c}{ GM(1,1) with Optimized } \\
GM(1,1) & $\begin{array}{l}\text { latest initial GM(1,1) } \\
\text { model }\end{array}$ & condition & model \\
\hline 2007 & $-20.3853 \%$ & $-11.4826 \%$ & $-6.4223 \%$ \\
2008 & $-16.7373 \%$ & $-7.9650 \%$ & $-1.8908 \%$ \\
2009 & $-13.9122 \%$ & $-5.3955 \%$ & $1.6908 \%$ \\
2010 & $-17.0273 \%$ & $-9.3490 \%$ & $-1.7448 \%$ \\
\hline
\end{tabular}

Where relative error is $r e l=\frac{\hat{y}_{i}-y_{i}}{y_{i}}, \hat{y}_{i}$ is prediction varue, $y_{i}$ is true value.

From Table 5, we can clearly ind that the optimized $\operatorname{GM}(1,1)$ model has the best performance in the relative error of three methods. The prediction accuracy is improved in each year. So maybe the fitting accuracy or optimized GM $(1,1)$ model is not very ideal in the beginning. But it can catcl the change tendency of the data better in the future. So the optimized $\operatorname{GM}(1,1)$ model based on least absolute criteria can provide a higher precision of prediction.

\section{Conclusion}

In this paper, we propose an optimized $\operatorname{GM}(1,1)$ model based on least absolute criteria. In the original GM(1, ) model, the initial condition is not very suitable because the fitting curve doesn't have to pass through the initial point. And we use the modified latest data which generating from the accumulative generating operation as the new initial condition. Though least square criteria are easy to calculate and widely used, it is not very reliable and will lead the accuracy of prediction to expand. So the least absolute criteria are used to overcome the problem. In addition, PSO algorithm is applied to the parameters optimization. At the end, the prediction results show that the optimized $\operatorname{GM}(1,1)$ model based on least absolute criteria improves the prediction accuracy by comparing with the original model and the $\operatorname{GM}(1,1)$ model with latest initial condition.

There also exist some improvements, such as improvement of background value and a better way to improve initial condition etc. These problems will be researched in the future in order to enhance the prediction accuracy. 


\section{Acknowledgements}

This research is conducted with the support of National Natural Science Foundation of China(71071045) and the national key project of "863" plan(2011AA05A116).

\section{References}

[1] D. Zafer and C. H. Lester, "Industrial electricity demand for Turkey: A structural time series analysis", Energy Economics, vol. 33, no. 3, (2011), pp. 426-436.

[2] A. Azadeh, M. Saberi, A. Gitiforouz and Z. Saberi, "A hybrid simulation-adaptive network based fuzzy inference system for improvement of electricity consumption estimation", Expert Systems with application, vol. 36, no. 8, (2009), pp. 11108-11117.

[3] K. Kavaklioglu, "Modeling and prediction of Turkey's electricity consumption using Support Vector Regression", Applied Energy, vol. 88, no. 1, (2011), pp. 368-375.

[4] K. F. TSO Geoffrey and K. W. Yau Kelvin, "Predicting electricity energy consumption: A comparison of regression analysis, decision tree and neural networks", Energy, vol. 32, no. 9, (2007), pp. 1761-1768.

[5] D. Julong, "Control problems of grey systems", Systems \& Control Letters, vol. 1, no. 5, (1982), pp. 288294.

[6] X. Xinping, G. Huan and M. Shuhua, "The modeling mechanism, extension and op mization of grey GM (1, 1) model”, Applied Mathematical Modelling, vol. 38, no. 5-6, pp 1896-1910.

[7] Z. Wei and H. JianMin, "Generalized GM $(1,1)$ model and its application in forecasting of fuel production", Applied Mathematical Modelling, vol. 37, no. 9, pp. 6234-6243.

[8] H. Changjun, T. Zhengqi, Z. Qingshan and C. Yuanzhi, "Application of Improved Error GM $(1,1)$ Model on Predicting of Cultivated Land in Yiyang”, Energy Procedia, vol. 5, pp. 1172-1\$76.

[9] T. Tzuli, "A research on the grey prediction model GM(1,n)", Applied Mathematics and Computation, vol. 218, no. 9, pp. 4903-4916.

[10] Z. Dahai, J. Shifang and S. Kaiquan, "Theoretical Defect of Grey Prediction Formula and Its Improvement", Systems Engineering-theory \& Practice, vol. 22, no. 8, (2002), pp 140-142.

[11] W. Yuhong, D. Yaoguo and L. Yueqing, "An approach to increase prediction precision of $\operatorname{GM}(1,1)$ model based on optimization of the initial condition", Expert Systems with Applications, vol. 37, no. 8, (2010), pp. $5640-5644$.

[12] S. Chisheng, H. Yentseng and J. Yeh, "Grey number prediction using the grey modification model with progression technique", Applied Mathematical Modelling, vol. 35, no. 3, (2011), pp. 1314-1321.

[13] W. Zhengxin, D. Yaoguo and I. Sifeng, "Optimization of Background Value in GM(1,1) Model", Systems Engineering - Theory \& Practice, vol. 28, no. 2, (2008), pp. 61-67.

[14] W. Xiaojia, "Application Research on Electricity Demand Forecasting Based on Gaussian Quadrature Formula", Procedia Engineering, vol. 15, (2011), pp. 5574-5578.

[15] Y. Tianxiang, L. Sifeng and X. Naiming, "On the properties of small sample of GM(1,1) model", Applied Mathemat cal Modêlling, vol 33, no. 4, (2009), pp. 1894-1903.

[16] J. Kennedy and R. C. Eberhart, "Particle swarm optimization", Proc. IEEE International Conference on Neural Networks, Perth. Austrália, vol. 4, (1995), pp. 1942-1948.

[17] H. Chechiang and C. Chiayon, "Applications of improved grey prediction model for power demand forecasting”, Energy Conversion and Management, vol. 44, no. 14, (2003), pp. 2241-2249.

[18] L. Chintsai and Y. Shihyu, "Forecast of the output value of Taiwan's opto-electronics industry using the Grey forecasting model", Technological Forecasting and Social Change, vol. 70, no. 2, (2003), pp. 177-186.

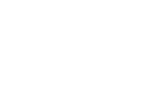

\section{Authors}

Zhang Zheng, he was born in 1987 and pursues a doctorate of management science and engineering at school of management of Hefei University of Technology in China. His research interests are prediction and data mining.

Yang Shanlin, he was born in 1948. He is a professor and Ph.D. supervisor in school of management of Hefei University of Technology in China. His research work is mainly engaged in the field of decision science, artificial intelligence, information management and information system. 
Liu Huizhou, he was born in 1985 and received his master degree from school of management of Hefei University of Technology in China in 2012. He is an engineer of Tongling Power Supply Company and his research interests are Power information system and computer network.

Yu Bengong, he was born in 1971. He is an associate professor in school of management of Hefei University of Technology in China. His research interests are computer network, information system, data structure and database.

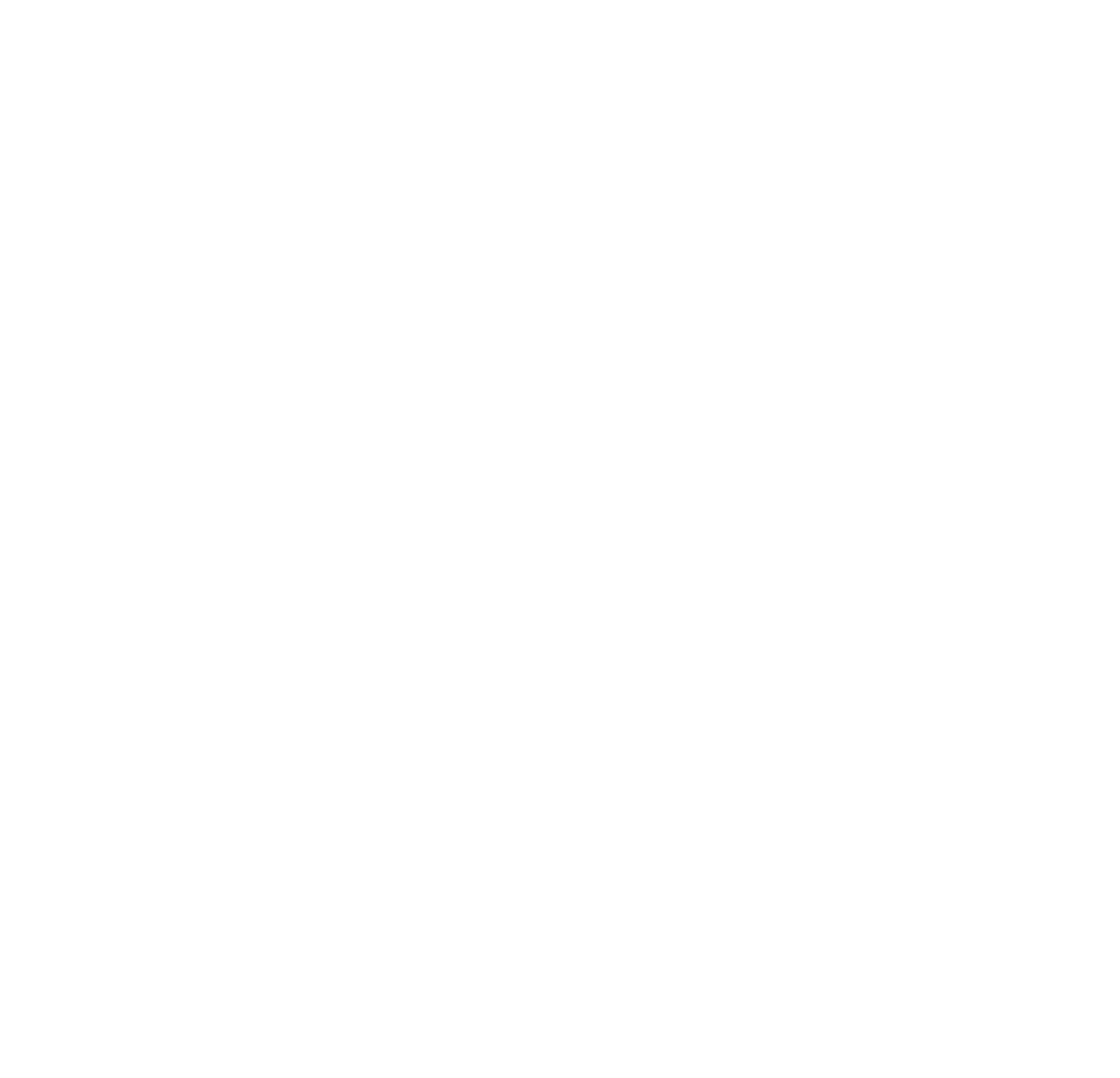


International Journal of Smart Home

Vol.8, No.4 (2014)

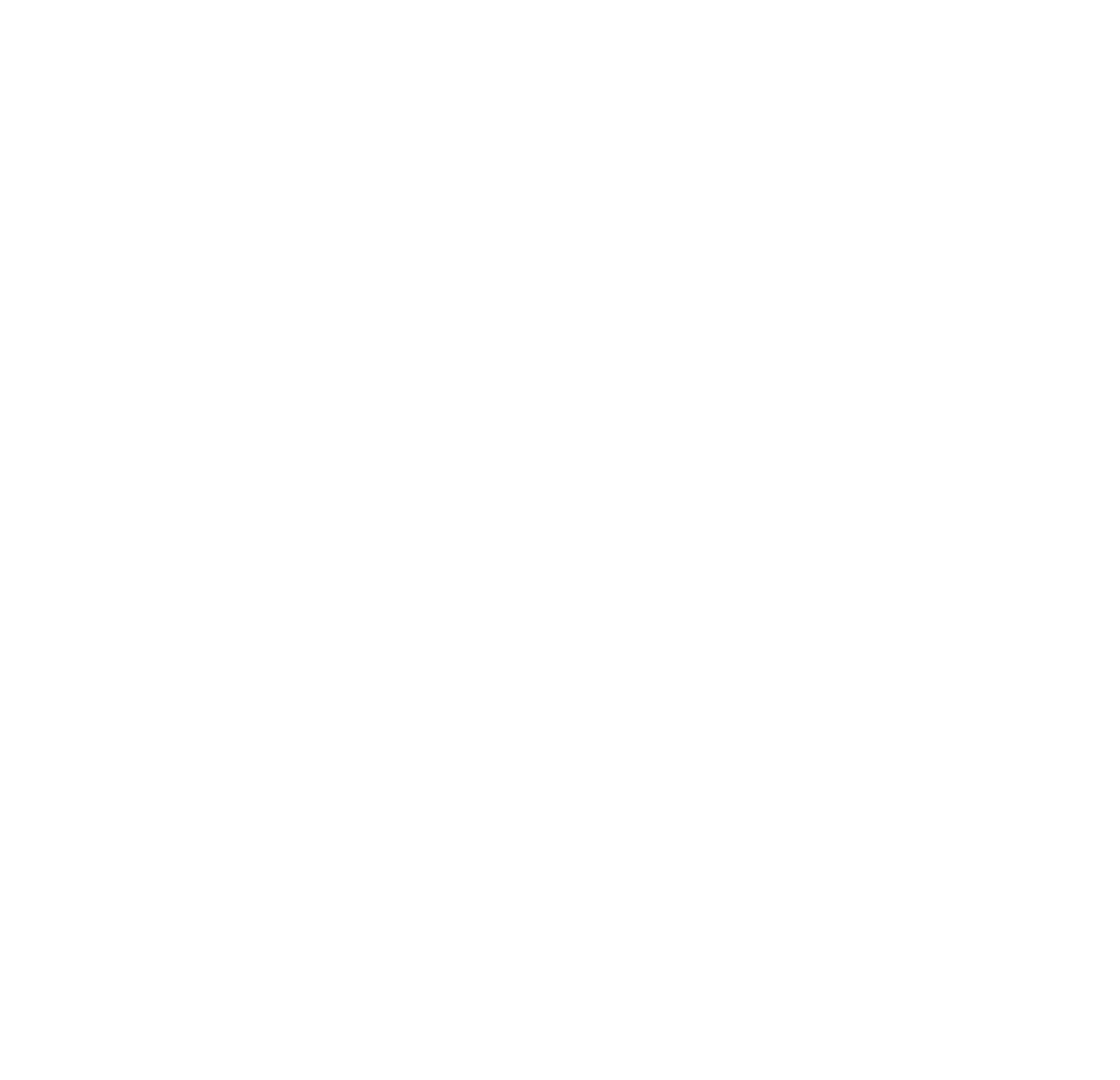

